\title{
Risk Factors and Patterns of Abdominal Lymph Node Recurrence After Radical Surgery for Locally Advanced Thoracic Esophageal Squamous Cell Cancer
}

\author{
Yichun Wang (DiD ${ }^{1, *}$ \\ Dongmei $\mathrm{Ye}^{2, *}$ \\ Mei Kang' \\ Liyang Zhu' \\ Shuhao Pan' \\ Fan Wang (1D
}

'Department of Radiation Oncology, The First Affiliated Hospital of Anhui Medical University, Hefei 230022, Anhui, People's Republic of China; ${ }^{2}$ Department of Thoracic Surgery, The First Affiliated Hospital of Anhui Medical University, Hefei 230022, Anhui, People's Republic of China

*These authors contributed equally to this work
This article was published in the following Dove Press journal:

Cancer Management and Research

Background: We aimed to identify the suitable indication and delineate the target volume based on the pattern of abdominal lymph node recurrence (ALNR) after radical surgery for guiding postoperative radiotherapy in thoracic esophageal squamous cell cancer (TESCC). Methods: Clinical data of patients with locally advanced TESCC after radical surgery without perioperative anti-tumor therapies from June 2011 to June 2016 were reviewed. Logistic regression analysis was used to find out the high-risk factors of ALNR. The pattern of ALNR was analysed and a template CT in the Pinnacle treatment plan system was used to reconstruct the distribution of the sites of ALNR.

Results: A total of 63 (19.57\%) patients with 276 lymph nodes of ALNR were identified in 322 patients. Univariate logistic regression indicated that pathological tumor location, width of tumor, T stage, $\mathrm{N}$ stage, TNM stage, ratio of lymph node metastasis (LNM), vessel carcinoma embolus, cancerous node, LNM in the middle and lower mediastinum, LNM in the abdominal region, ratio of LNM in the abdominal region were risk factors of ALNR. Multivariate logistic regression analysis showed that only LNM in the abdominal region was an independent risk factor. The odds ratio was 7.449 (95\% $\mathrm{CI}=2.552-22.297, \mathrm{P}<0.001)$. Station $16 \mathrm{a} 2$, station 9 , station 16b1, and station 8 were the major regions of ALNR. The recurrence rates were $10.56 \%$, $9.63 \%, 7.14 \%$ and $5.28 \%$ in these stations, respectively.

Conclusion: Positive pathological abdominal lymph nodes should be the major indication for abdominal irradiation in postoperative radiotherapy for locally advanced TESCC. We recommended that the target volume includes station 8 , station 9, station $16 \mathrm{a} 2$ and station $16 \mathrm{~b} 1$ and proposed a specific delineation of the clinical target volume based on the distribution of ALNR on template CT images.

Keywords: esophageal carcinoma, clinical target volume, recurrence, postoperative radiotherapy, abdominal lymph nodes

\section{Background}

As the 6th most common cause of cancer-related death worldwide, esophageal cancer (EC) is still a global health problem. Due to the poor survival associated with surgery alone, multidisciplinary treatments have been studied worldwide for locally advanced EC. ${ }^{1}$ Neoadjuvant chemoradiotherapy followed by surgery is recommended as a standard strategy. However, a large number of patients choose upfront surgery in the real world, ${ }^{2,3}$ especially in China which includes nearly half of all cases diagnosed and 
wherein esophageal squamous cell cancer (ESCC) accounts for more than $90 \%{ }^{4}$ Adjuvant therapies should be considered for clinical early-stage $\mathrm{EC}$ with a high risk or upstaged tumors after surgery, or patients with locally advanced EC who received upfront surgery. Adjuvant chemoradiotherapy after surgery has shown a significant survival benefit for locally advanced EC according to many studies, ${ }^{5}$ including the only existing randomized controlled trial. ${ }^{6}$ Therefore, adjuvant chemoradiotherapy is also carried out in clinical practice for some locally advanced EC.

Due to the complex lymphatic drainage system of the esophagus, it is difficult to design a suitable target volume for radiotherapy of thoracic EC (TEC). ${ }^{7}$ Many target volumes of postoperative radiotherapy (PORT) have been used in past decades without an existing standard one, ranging from a small field consisting of only the primary tumor bed to a large field consisting of the supraclavicular, mediastinal and upper abdominal regions. Designing a more reasonable target volume for PORT is desperately necessary. It may not only improve the efficacy but also decrease the complications in TEC. Our retrospective analysis suggested that the supraclavicular and upper mediastinal regions had high recurrence rates for all TEC, while the abdominal region had a high recurrence rate for the lower thoracic ESCC (TESCC). ${ }^{8,9}$ These results were similar to those of a pooled analysis of published results. ${ }^{10}$ The supraclavicular and upper mediastinal regions were recommended to be included in the target volume of PORT for TEC. However, there is little consensus on which patients and irradiation fields are suitable for the PORT in the abdominal area. The pooled analysis indicated that the irradiation field should include the abdominal paraaortic lymph nodes (LNs) for lower TESCC while the upper abdominal LNs might not necessarily be included. $^{10}$ Another study suggested that the abdominal area seemed to be an elective irradiation target for lower TESCC with pathological stage IIIB or higher. ${ }^{11}$ Although there was a retrospective study proposed an abdominal target volume for the PORT of TESCC, the incorrect illustration of many areas of the abdominal lymph node recurrence (ALNR) made this result unreliable. ${ }^{12}$ Another study using a lymph node map of esophageal cancer based on the 8th edition of AJCC was insufficient for analysis of the detail distribution of ALNR. ${ }^{11}$

In this study, we reviewed the data of patients with locally advanced TESCC who received initial esophagectomy without adjuvant anti-tumor therapies before a recurrence in our hospital. The patterns and risk factors of
ALNR were analysed to identify the suitable patients and irradiation fields for PORT in locally advanced TESCC.

\section{Patients and Methods}

\section{Patients}

Clinical data of patients seen from June 2011 to June 2016 were reviewed at the First Affiliated Hospital of Anhui medical University. Clinical pathological characteristics (tumor invasion, node status, metastasis, and stage) were recorded based on the TNM classification (8th edition) according to the International Union Against Cancer.

\section{Patient Selection}

The inclusion criteria were as follows: 1) patients with TEC without clinical distant metastasis before surgery; 2) patients with pathological $\mathrm{T} 2-4 \mathrm{aN} 0$ or $\mathrm{T} 1-4 \mathrm{aN}+$ diseases; 3) Patients who had not received anti-tumor therapies before surgery; 4) patients who received radical esophagectomy and 2-field or 3-field lymphadenectomy; 5) patients with pathologically confirmed ESCC; 6) patients had not received chemotherapy, radiotherapy or other anti-tumor therapies before recurrence; and 7) patients with a followup time of more than 2 years. The exclusion criteria were as follows: 1) patients with cervical EC; 2) patients with unknown or unclear pathological records; 3) patients with double or multiple primary cancers; and 4) patients with uncertain recurrence sites.

\section{Regional Lymph Node Categorization}

The names and numbers of regional node divisions in the abdominal region were based on the Japanese Classification of Gastric Cancer. ${ }^{13}$ Due to the anatomic changes after surgery, only LNs within station 7 to station 20 were used in this study. The classification standards were as follows: station 7 (LNs along the left gastric artery), station 8 (LNs along the common hepatic artery), station 9 (LNs along the celiac artery), station 10 (LNs at the splenic hilum), station 11 (LNs along the splenic artery), station 12 (LNs in the hepatoduodenal ligament), station 13 (LNs on the posterior surface of the pancreatic head), station 14 (LNs along the superior mesenteric vessels), station 15 (LNs along the middle colic artery), station 16 (16a1, paraaortic LNs in the diaphragmatic aortic hiatus; 16a2, paraaortic LNs between the upper margin of the origin of the celiac artery and the lower border of the left renal vein; 16b1, paraaortic LNs between the lower border of the left renal vein and the upper border of the origin of the inferior mesenteric artery; 
16b2, paraaortic LNs between the upper border of the origin of the inferior mesenteric artery and the aortic bifurcation), station 17 (LNs on the anterior surface of the pancreatic head), station 18 (LNs along the inferior margin of the pancreas), station 19 (infradiaphragmatic LNs) and station 20 (LNs in the esophageal hiatus of the diaphragm).

\section{Diagnosis of Recurrence}

Regional lymph node recurrence was diagnosed mainly based on computed tomography (CT) images, occasionally magnetic resonance (MR) images or positron emission tomography (PET)/CT images. LNs with a short-axis diameter of over $8.00 \mathrm{~mm}$ in the abdomen on CT/MR images or LNs with fusion or necrosis were considered to represent ALNR, and LNs with a SUVmax value more than 2.4 on PET/CT images were considered to represent ALNR.

\section{Delineation of Recurrent abdominal Lymph Nodes on Template CT}

We selected a 70-year-old woman with middle thoracic ESCC who underwent esophagectomy in the real world as a standard patient. This patient met the following criteria: (1) lymphadenectomy including stations 1-4, station 7, station 8 and station 9 were carried out; (2) no identified ALNR; (3) no obvious abnormalities in organs and structures in the abdominal region; and (4) performance of contrast-enhanced CT scanning of a $0.62 /$ $0.63 \mathrm{~mm}$ thick section from the cricoid cartilage to 5.00 $\mathrm{cm}$ below the iliac bifurcation. The template CT images were imported into the Pinnacle 3 treatment planning system (version 9.8.0.6007; Philips Medical Systems, Fitchburg, WI, USA) for the delineation and reconstruction of LNs.

All the locations of ALNR were transferred to the corresponding anatomic positions in the template CT images by two radiation oncologists and a radiologist. The anatomic positions were mainly referred to the surrounding vascular and skeletal structures. All LNs were plotted with a diameter of $2.00 \mathrm{~mm}$ according to their geometric centre. When there were mixed LNs, we plotted the geometric centre of each node that was distinguishable in its respective location. Otherwise, we plotted a geometric centre for the mixed lymph node. After the areas of ALNR of all the patients were transferred to the template CT images, the merged target volume was expanded outward by $3.00 \mathrm{~mm}$ and expanded $4.00 \mathrm{~mm}$ on the upper and lower bounds for all these plotted LNs.

\section{Follow-Up}

Follow-up after surgery was conducted every 2-3 months for the first 6 months, every 3-4 months thereafter within the first 2 years and every 6 months after 2 years. Chestenhanced CT, abdominal and cervical ultrasound or enhanced CT was implemented for re-examinations. When a suspicious or an enlarged lymph node was found by ultrasound, CT, MR or PET/CT was performed. The cut-off for follow-up was June 2019.

\section{Statistical Analysis}

The statistical package SPSS (version 22.0 for Windows, IBM SPSS, Armonk, NY, USA) was used for statistical analysis. For categorical variables, the chi-square test was used. Logistic regression analyse were performed to analyze the hazard ratio of risk factors. A value of $\mathrm{P}<0.05$ was used as the significance threshold.

\section{Results}

\section{Characteristics of the Included Patients}

After the systematic screening, there were a total of 322 patients included in our analysis. The median follow-up time was 42 months (range, 26 to 89 months). A total of $63(19.57 \%)$ patients were identified as having ALNR until the last follow-up time. The general information for these patients is shown in Table 1. There were 252 $(78.26 \%)$ male patients. The median age was 64 years old (range, 39 to 82 years old). The primary tumors of $27(8.39 \%), 217(67.39 \%)$ and $78(24.22 \%)$ patients were located in the upper, middle and lower thoracic esophagus, respectively. Left transthoracic esophagectomy and the thoracoscopy and laparoscopic combined esophagectomy were the major operation methods ( $84.16 \%$ of patients). Two-field lymphadenectomy was carried out for $95.96 \%$ (309/322) of patients. The median number of resected LNs was 14 (range, 4 to 66) and 175 (54.34\%) patients had negative pathological lymph node metastasis. Of 63 patients with ALNR, 15 (23.81\%) exhibited no recurrence in other sites, $21(33.33 \%)$ had supraclavicular or/and mediastinal lymph node recurrence without hematological recurrence, and $27(42.86 \%)$ developed hematological recurrence (Table 2).

\section{Risk Factors for ALNR}

The risk factors for ALNR were analysed by univariate and multivariate logistic regression analyses. As shown in Table 3, tumor location, width of tumor, T stage, N stage, TNM stage, 
Table I Characteristics of the Included Patients

\begin{tabular}{|c|c|c|c|}
\hline Pathological Parameters & Values & Cases of ALNR & Rate of ALNR \\
\hline \multicolumn{4}{|l|}{ Gender } \\
\hline Male/female & $252 / 70$ & $51 / 12$ & $20.24 \% / 17.14 \%$ \\
\hline Age (years) & $63.93 \pm 7.64$ & 63 & $19.57 \%$ \\
\hline \multicolumn{4}{|l|}{ Location } \\
\hline Upper/middle/lower & $27 / 217 / 78$ & $2 / 34 / 27$ & $7.41 \% / 15.67 \% / 34.62 \%$ \\
\hline Length $(\mathrm{cm})$ & $3.90 \pm 1.43$ & 63 & $19.57 \%$ \\
\hline Width $(\mathrm{cm})$ & $2.54 \pm 0.99$ & 63 & $19.57 \%$ \\
\hline \multicolumn{4}{|l|}{ T stage } \\
\hline $\mathrm{TI} / \mathrm{T} 2 / \mathrm{T} 3 / \mathrm{T} 4 \mathrm{a}$ & $10 / 98 / 206 / 8$ & $3 / 1 \mathrm{I} / 44 / 5$ & $30.00 \% / 11.22 \% / 21.36 \% / 62.50 \%$ \\
\hline \multicolumn{4}{|l|}{$\mathrm{N}$ stage } \\
\hline No/NI/N2/N3 & $175 / 108 / 25 / 14$ & $|1 / 28 /| 3 /|1|$ & $6.29 \% / 25.93 \% / 52.00 \% / 78.57 \%$ \\
\hline \multicolumn{4}{|l|}{ TNM stage } \\
\hline II A/ II B/IIIIA/IIIB/IVA & $73 / 112 / 26 / 88 / 23$ & $3 / 11 / 7 / 26 / 16$ & $4.11 \% / 9.82 \% / 26.92 \% / 29.55 \% / 69.57 \%$ \\
\hline \multicolumn{4}{|l|}{ Differentiation } \\
\hline Poor/moderate/well & $92 / 212 / 18$ & $23 / 36 / 4$ & $25.00 \% / 16.98 \% / 22.22 \%$ \\
\hline \multicolumn{4}{|l|}{ Operation method } \\
\hline LT/RT/TLC/other & $119 / 47 / 152 / 4$ & $30 / 24 / 9 / 0$ & $25.21 \% / 51.06 \% / 5.92 \% / 0.00 \%$ \\
\hline Number of resected LNs & $15.22 \pm 8.76$ & 63 & $19.57 \%$ \\
\hline \multicolumn{4}{|l|}{ Fields of lymphadenectomy } \\
\hline Two/three & $309 / 13$ & $60 / 3$ & $19.42 \% / 23.08 \%$ \\
\hline \multicolumn{4}{|l|}{ Vessel carcinoma embolus } \\
\hline Yes/no & $27 / 295$ & $11 / 52$ & $40.74 \% / 17.63 \%$ \\
\hline \multicolumn{4}{|l|}{ Cancerous node* } \\
\hline Yes/no & $17 / 305$ & $7 / 56$ & $41.18 \% / 18.36 \%$ \\
\hline
\end{tabular}

Note: *Extranodal metastasis near the primary tumor.

Abbreviations: ALNR, abdominal lymph node recurrence; LNs, lymph nodes; LT, left transthoracic esophagectomy; N, node; RT, right transthoracic esophagectomy; T, tumor; TLC, thoracoscopy and laparoscopic combined esophagectomy; TNM, tumor-node-metastasis.

Table 2 The Relationship Between ALNR with Recurrence in Other Sites

\begin{tabular}{|c|c|c|c|c|}
\hline Recurrence Site & Upper TEC & Middle TEC & Lower TEC & TEC \\
\hline ALNR only & $0(0.00 \%)$ & $6(17.65 \%)$ & $9(33.33 \%)$ & $15(23.81 \%)$ \\
\hline ALNR+ supraclavicular LNR & $0(0.00 \%)$ & I (2.9l\%) & $4(14.81 \%)$ & $5(7.94 \%)$ \\
\hline ALNR+ mediastinal LNR & I (50.00\%) & $6(17.65 \%)$ & $4(\mid 4.81 \%)$ & II (17.46\%) \\
\hline ALNR+ hematological R & $0(0.00 \%)$ & 7 (20.59\%) & $5(18.52 \%)$ & $12(19.05 \%)$ \\
\hline ALNR+ supraclavicular+ mediastinal LNR & I (50.00\%) & $4(11.76 \%)$ & $0(0.00 \%)$ & $5(7.94 \%)$ \\
\hline ALNR+ supraclavicular LNR+ hematological $R$ & $0(0.00 \%)$ & $3(8.82 \%)$ & $0(0.00 \%)$ & $3(4.76 \%)$ \\
\hline ALNR+ mediastinal LNR+ hematological $R$ & $0(0.00 \%)$ & $6(17.65 \%)$ & 4 (14.8I\%) & $10(15.87 \%)$ \\
\hline$A L N R+$ supraclavicular $L N R+$ mediastinal $L N R+$ hematological $R$ & $0(0.00 \%)$ & I (2.9l\%) & I (3.70\%) & $2(3.17 \%)$ \\
\hline Total & $2(100.00 \%)$ & $34(100.00 \%)$ & $27(100.00 \%)$ & $63(100.00 \%)$ \\
\hline
\end{tabular}

Abbreviations: ALNR, abdominal lymph node recurrence; LNR, lymph node recurrence; R, recurrence; TEC, thoracic esophageal cancer. 
Table 3 Logistic Regression Analysis of Risk Factors on ALNR

\begin{tabular}{|c|c|c|c|c|c|c|}
\hline \multirow[t]{2}{*}{ Parameters } & \multicolumn{3}{|c|}{ Univariate Analysis } & \multicolumn{3}{|c|}{ Multivariate Analysis } \\
\hline & OR & $95 \% \mathrm{Cl}$ & $P$ value & OR & $95 \% \mathrm{Cl}$ & $P$ value \\
\hline Gender (Female vs male) & 1.226 & $0.613-1.226$ & 0.564 & - & - & - \\
\hline Age (years) & 0.987 & $0.952-1.023$ & 0.463 & - & - & - \\
\hline \multicolumn{7}{|l|}{ Location } \\
\hline (Middle vs upper) & 2.322 & $0.525-10.264$ & 0.266 & 0.975 & $0.185-5.143$ & 0.976 \\
\hline (Lower vs upper) & 6.618 & I.456-30.074 & 0.014 & $2.38 I$ & $0.435-13.018$ & 0.317 \\
\hline Length (cm) & 1.086 & $0.899-1.312$ & 0.394 & - & - & - \\
\hline Width $(\mathrm{cm})$ & 1.399 & $1.075-1.820$ & 0.013 & 1.180 & $0.823-1.692$ & 0.367 \\
\hline T stage (TI/T2/T3/4a) & 1.852 & $1.090-3.146$ & 0.023 & 1.584 & $0.631-3.978$ & 0.327 \\
\hline N stage (N0/NI/N2/N3) & 3.911 & $2.664-5.743$ & $<0.001$ & 2.004 & $0.700-5.734$ & 0.195 \\
\hline TNM stage ( II a/ II b/IIIa/IIIb/IVa) & 2.335 & $1.804-3.021$ & $<0.001$ & 0.693 & $0.312-1.538$ & 0.367 \\
\hline Differentiation (Well/moderate/poor) & 0.735 & $0.440-1.226$ & 0.238 & - & - & - \\
\hline \multicolumn{7}{|l|}{ Operation method } \\
\hline (LT vs TLC) & 1.027 & $0.564-1.872$ & 0.930 & - & - & - \\
\hline (RT vs TLC) & 0.963 & $0.420-2.207$ & 0.929 & - & - & - \\
\hline (Other vs.TLC) & - & - & - & - & - & - \\
\hline Number of resected LNs & 0.991 & $0.962-1.022$ & 0.584 & - & - & - \\
\hline Ratio of LNM & 301.618 & $47.720-1906.377$ & $<0.001$ & 1.899 & $0.095-37.805$ & 0.674 \\
\hline Lymphadenectomy (Three vs two fields) & 1.245 & $0.332-4.664$ & 0.745 & - & - & - \\
\hline Vessel carcinoma embolus (Yes vs no) & 3.213 & I.409-7.324 & 0.006 & 2.900 & $0.974-8.638$ & 0.056 \\
\hline Cancerous node (Yes vs no) & 3.112 & $1.135-8.532$ & 0.027 & 0.712 & $0.197-2.574$ & 0.604 \\
\hline \multicolumn{7}{|l|}{ Regional LNs } \\
\hline UM (Positive vs negative) & 1.947 & $0.805-4.708$ & 0.139 & - & - & - \\
\hline MLM (Positive vs negative) & 3.305 & $|.8| 1-6.03 \mid$ & $<0.001$ & 1.662 & $0.614-4.501$ & 0.318 \\
\hline AR (Positive vs negative) & 12.900 & $6.840-24.329$ & $<0.001$ & 7.499 & $2.522-22.297$ & $<0.001$ \\
\hline Resected number of $A R$ & 1.032 & $0.976-1.091$ & 0.273 & - & - & - \\
\hline Ratio of LNM in AR & 127.684 & $28.529-57|.46|$ & $<0.001$ & 2.215 & $0.284-15.895$ & 0.463 \\
\hline
\end{tabular}

Note: Bold text indicates a statistical significant $(P<0.05)$.

Abbreviations: AR, abdominal region; ALNR, abdominal lymph node recurrence; Cl, confidence interval; LNs, lymph nodes; LNM, lymph node metastasis; LT, left transthoracic esophagectomy; MLM, middle and lower mediastinum; N, node; OR, odds ratio; RT, right transthoracic esophagectomy; T, tumor; TLC, thoracoscopy and laparoscopic combined esophagectomy; TNM, tumor-node-metastasis; UM, upper mediastinum.

ratio of lymph node metastasis (LNM), vessel carcinoma embolus, cancerous node, LNM in the middle and lower mediastinal region, LNM in the abdominal region, and ratio of LNM in the abdominal region at the time of surgery were risk factors for ALNR. After multivariate logistic regression analysis using all these risk factors, only LNM in the abdominal region was an independent risk factor for ALNR. The odds ratio (OR) was 7.449 (95\% confidence interval $=2.552-22.297$, $\mathrm{P}<0.001)$.

\section{Patterns of ALNR}

A total of 276 LNs associated with ALNR in 63 patients were identified. The ratio of recurrent LNs (the number of recurrent LNs in each station to the total number of recurrent LNs) and the recurrence rates of different stations in the abdominal region are shown in Figure 1. Station 16a2 and station 9 were the major regions of ALNR, followed by station 16b1 and station 8 . Their recurrence rates of TEC were $10.56 \%$, $9.63 \%, 7.14 \%$, and $5.28 \%$ in these stations, respectively, and 


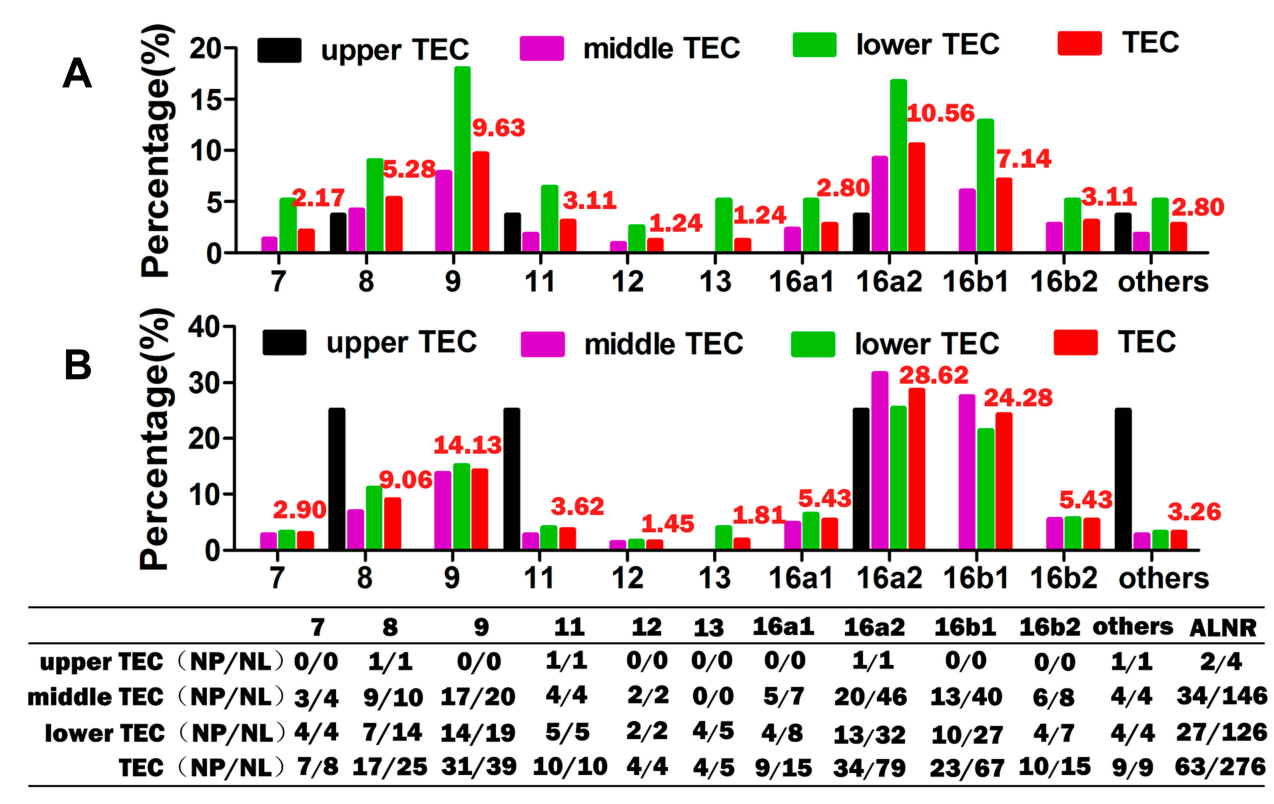

Figure I ALNR in different lymph node stations after radical esophagectomy. (A) recurrence rate; (B) ratio of recurrent LNs.

Abbreviations: ALNR, abdominal lymph node recurrence; LNs, lymph nodes; NP/NL, number of patients/number of lymph nodes; TEC, thoracic esophageal cancer.

the total number of recurrent LNs in these four stations accounted for $76.09 \%$ (210/276) of the overall recurrent LNs.

\section{Mapping of ALNR on the Template CT}

The three-dimensional reconstructed distribution of all recurrent LNs using the merged target volume is shown in Figure 2. Although ALNR was found in a wide region from the subphrenic to the iliac bifurcation, it mainly surrounded the abdominal aorta (AA) and its main branches, including the celiac artery (CA) and the common hepatic artery (CHA). Only a few recurrent LNs were found around the splenic artery and at the splenic hilus. Moreover, ALNR was mainly located at the left and anterior side of the AA, partly between the AA and the inferior vena cava (IVC), and there was no ALNR located on the right side of the IVC.

Figure 3 shows the areas of ALNR on the cross-sectional template CT images in $5.00 \mathrm{~mm}$ thick sections (every 8 slices). ALNR in station 9 and station 8 mainly surround the AA and the CHA. ALNR in station 16a2 and station $16 \mathrm{~b} 1$ was mainly located on the anterior and bilateral side of the AA. ALNR between the AA and IVC occurred from approximately $1.00 \mathrm{~cm}$ above the upper side of the CA to approximately $2.75 \mathrm{~cm}$ below the lower side of the left renal vein (LRV) in our study. ALNR in station 16b1, it was generally located on the bilateral side of the AA below the upper side of the horizontal part of the duodenum (approximately $0.50 \mathrm{~cm}$ below the lower side of the LRV in our study).

\section{Discussion}

The esophagus has a complex lymphatic drainage system, characterized by bidirectional drainage both up to the neck and down to the abdominal area. As a result, abdominal LNM is common for TEC. The most common abdominal regions for LNM of TEC are LNs along the left gastric artery, paracardial LNs and lesser curvature LNs, followed by LNs along the CA and LNs along the CHA. ${ }^{7}$ Abdominal lymph node dissection is recommended for TEC. However, ALNR is common for patients after radical surgery, especially for patients with lower TEC. It was reported that the rate of ALNR ranged from 9.3\% to $20.0 \%$ after surgery for TEC, ${ }^{12}$ and it was $19.57 \%$ in our study. Therefore, ALNR is one of the major occurrences in TEC after esophagectomy.

Postoperative radiotherapy/chemoradiotherapy can improve the survival and decrease rates of recurrence or distant metastasis for patients with locally advanced TEC after upfront surgery. ${ }^{14-16}$ However, which patients are suitable for irradiation of the abdominal region after surgery is controversial. In most previous studies, irradiation of the abdominal region was recommended for patients with lower TEC due to its high rate of ALNR. ${ }^{8,10,12}$ Although patients with lower TEC are most likely to benefit from PORT in the abdominal region, ALNR may 

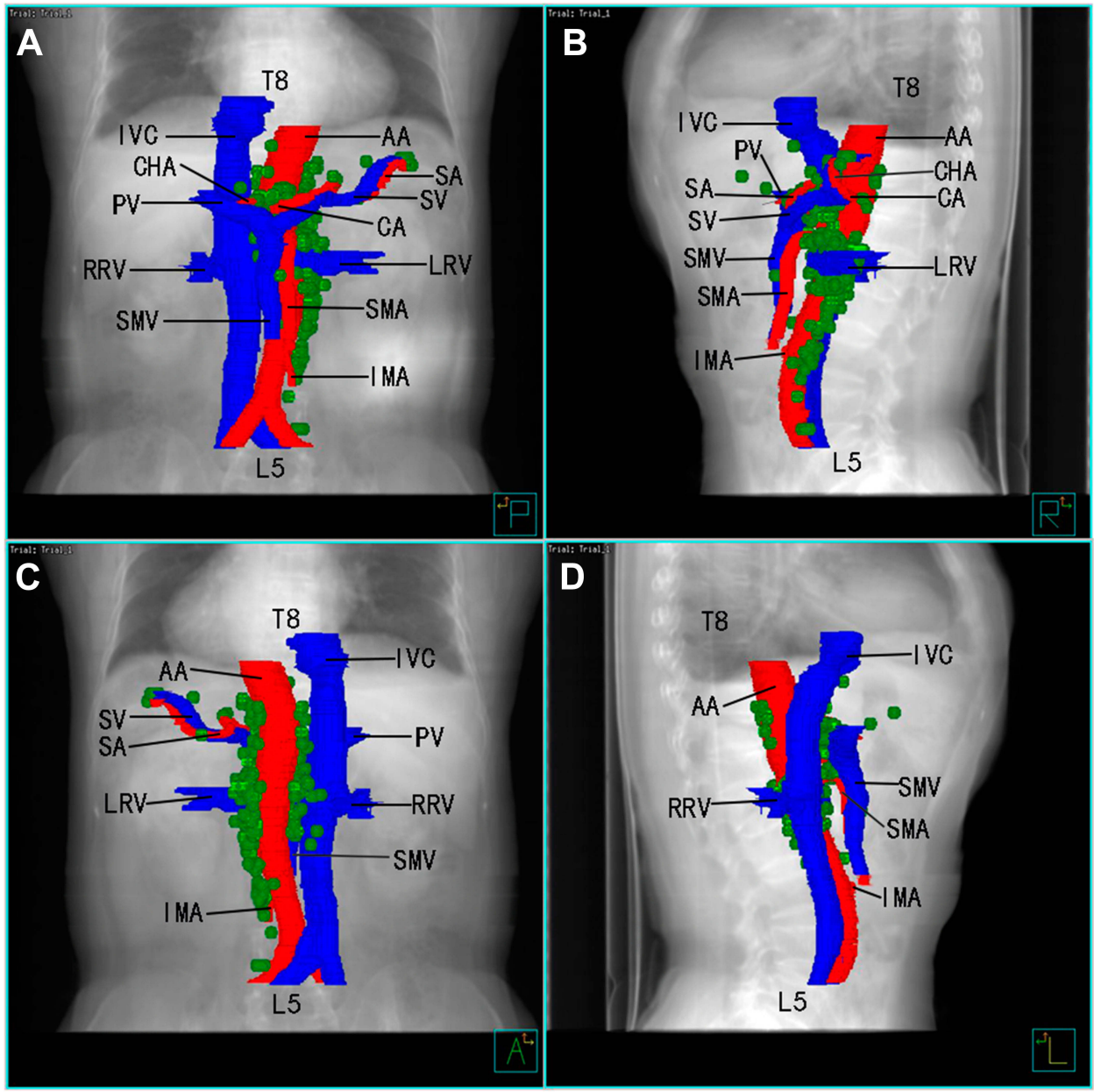

Figure 2 Three-dimensional reconstructed images of the major vessels and the merged target volume of recurrent abdominal LNs on the template CT. (A) anteriorposterior; (B) left-right; (C) posterior-anterior; (D) right-left.

Abbreviations: AA, abdominal aorta; CA, celiac artery; CHA, common hepatic artery; CT, computed tomography; IMA, inferior mesenteric artery; IVC, inferior vena cava; LN, lymph node; LRV, left renal vein; PV, portal vein; RRV, right renal vein; SA, splenic artery; SMA, superior mesenteric artery; SMV, superior mesenteric vein; SV, splenic vein; T8, 8th thoracic vertebra; L5, 5th lumbar vertebra.

be found in all TEC patients due to the bidirectional lymphatic drainage of the esophagus. ${ }^{7}$ In this study, we found that only LNM in the abdominal region was an independent factor for ALNR. Generally, the descending lymphatics of the esophagus are first relayed with the perigastric LNs (LNs along the left gastric artery, paracardial LNs and lesser curvature LNs). ${ }^{7}$ Radio-guided detection of sentinel lymph nodes (SLNs) in patients with preoperative T1N0M0 or T2N0M0 stage primary EC also showed that SLNs were mainly detected in these perigastric LNs. ${ }^{17}$ Therefore, LNM in the abdominal region at the time of surgery should be a good indicator of distant LNM in the abdominal region. Given these points, we recommend that pathological positive abdominal LNs be the major indication for abdominal irradiation in PORT for TEC.
A consensus on the topic of the target volume of PORT for TEC has not reached. In the abdominal area, LNs along the left gastric artery and paracardial LNs were included in the target volume in many studies ${ }^{17-19}$ while all LNs were omitted in other studies. ${ }^{20}$ There are three important factors we should consider when designing the irradiation field of PORT: (1) the anatomic lymphatic drainage of the esophagus; (2) the lymph node dissection of routine surgery, and (3) the pattern of recurrence after surgery. As stations with a high rate of LNM, the perigastric LNs first receive the downward lymphatics of the esophagus. ${ }^{7}$ However, these LNs can be cleared early by routine surgery and ALNR was rarely found in these primary stations. ${ }^{8,12}$ This was also confirmed in this study. The next major relayed LNs are LNs along the CA (station 9), LNs along the CHA (station 8) and the LNs along the 

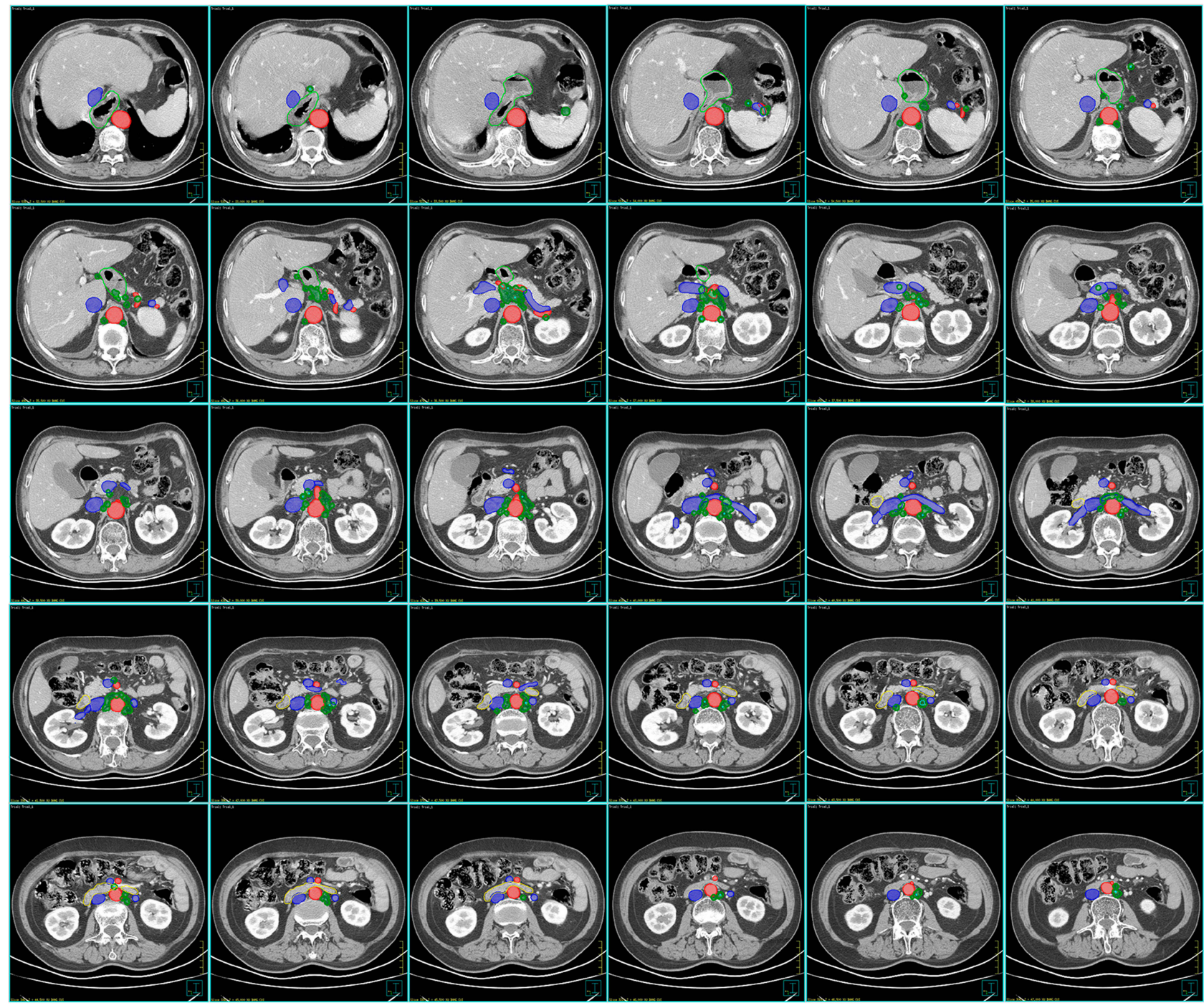

Figure 3 Consistency mapping of recurrent abdominal LNs on the template computed tomography images from the cranial to caudal direction in 5.00 mm thick sections (every 8 slices). Red lines: arteries; blue lines: veins; green lines: the merged target volume; sky blue lines: the plotted lymph nodes.

AA (station 16), which are positioned before the lymphatic drainage is collected in the cisterna chyli or thoracic duct. ${ }^{7}$ These LNs were not or not completely cleared by routine lymphadenectomy. As a result, these stations become the high-risk regions for ALNR. Yu et al reported that the number of recurrent LNs along the AA accounted for 57.14\% (20/35) recurrent LNs. ${ }^{11}$ Our present study showed that station $16 \mathrm{a} 2$, station 9, station $16 \mathrm{~b} 1$ and station 8 were the stations with high rates of ALNR (10.56\%, $9.63 \%, 7.14 \%$, and $5.28 \%$, respectively). Most abdominal lymphatics should be collected in the cisterna chyli which is located at the surface of the second lumbar vertebra (variably, the 12th thoracic vertebra to the second lumbar vertebra). ${ }^{21}$ This may be the major reason for the high rates of ALNR at stations $16 \mathrm{a} 2$ and $16 \mathrm{~b} 1$, which seemed to be similar to the high rates of postoperative lymph node recurrence in gastric cancer. ${ }^{22}$ Taken together, these foundings suggest that the target volume of PORT in the abdominal area for TESCC should mainly include station 16a2, station 9, station $16 \mathrm{~b} 1$ and station 8 .

Our study using template CT-based images also provides information for specific delineation of the clinical target volume. According to the distribution of ALNR, we proposed a clinical target volume including station 8 , station 9, station $16 \mathrm{a} 2$ and station $16 \mathrm{~b} 1$ for PORT of TESCC (Figure 4). The upper border should be more than $5.00 \mathrm{~mm}$ from the upper side of the CHA to include station 8 (Figure 4A). The lower border is the upper side of the inferior mesenteric artery (Figure $4 \mathrm{~L}$ ). The anterior border is the terminal end of the CHA, the posterior side of the pancreas and the stomach or the duodenum in the 

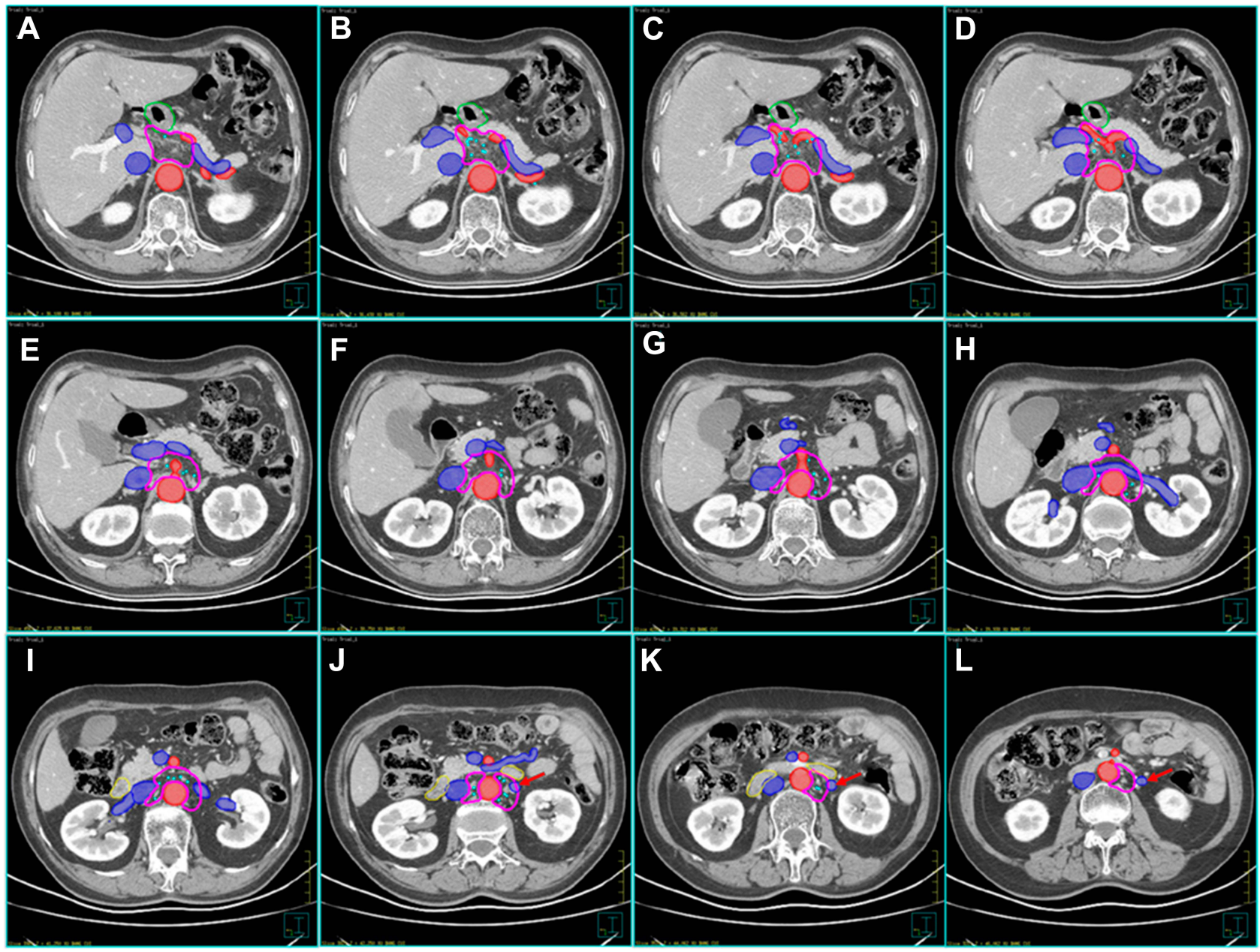

Figure 4 A proposed delineation of the target volume including station 8 , station 9 , station $16 \mathrm{a} 2$ and station $16 \mathrm{~b} 1$ in the abdominal area for postoperative radiotherapy of thoracic esophageal squamous cell cancer. (A) $5.00 \mathrm{~mm}$ above the upper side of the common hepatic artery; (B) the upper side of the common hepatic artery; (C) I.00 cm above the upper side of the origin of the celiac artery; (D) the terminal end of the celiac artery; (E) the upper side of the origin of celiac artery; (F) the upper side of the superior mesenteric artery; (G) the upper side of the origin of the superior mesenteric artery; $(\mathbf{H})$ the middle of the left renal vein; (I) the lower side of the left renal vein; (J) the upper side of the horizontal part of duodenum; (K) $2.75 \mathrm{~cm}$ below the lower of the left renal vein; (L) the upper side of the inferior mesenteric artery. Red lines: arteries; blue lines: veins; pink lines: the proposed target volume; sky blue lines: plotted lymph nodes; red arrow: left ovarian vein.

upper part (Figure 4A-E), the posterior of the pancreatic head and the superior mesenteric vessels (Figure 4F-I) in the middle part and the posterior of the horizontal part of the duodenum in the lower part (Figure 4J-L). The right border is the left side of the portal vein, liver, and the IVC in the upper part (Figure 4A$\mathrm{E}$ ), the left side of the pancreatic head and IVC in the middle part (Figure 4F-J) and the left side of the AA in the lower part (Figure 4K-L). The left border is approximately at the level of the left side of the vertebra in the upper part (Figure 4A-E) and the left ovarian vein (or testicular vein for man) in the lower part (Figure 4F-L). Due to the low recurrence rate and the lack of a single site of ALNR behind the crus of the diaphragm or at the posterior of the AA, we suggest that the AA may not be included in the target volume for the prophylactic PORT. Therefore, the posterior border is the surface of the crus of the diaphragm at the anterior of the $\mathrm{AA}$ or the anterior of the AA (Figure 4A-I), the posterior of the IVC on the right side of the AA from approximately $1.00 \mathrm{~cm}$ above the upper side of the origin of the CA to approximately $2.75 \mathrm{~cm}$ below the lower side of the LRV (Figure 4C-J) and the posterior side of the AA, and the anterior of the vertebra and psoas major muscle on the left side of the AA from the upper side of the origin of CA to the upper side of the inferior mesenteric artery (Figure 4E-L). From the upper side of the horizontal part of the duodenum (approximately $0.50 \mathrm{~cm}$ below the lower side of the LRV in our study), there was no ALNR at the anterior side of the AA, so the target volume could be separated into two segments on the bilateral sides of the AA to decrease the irradiation to the duodenum (Figure 4J). Then, from the level of approximately $2.75 \mathrm{~cm}$ below the lower side of the LRV, there was no ALNR 
in the interval between the IVC and the AA, so we include only the left side of station $16 \mathrm{~b} 1$ in the target volume (Figure $4 \mathrm{~K}-\mathrm{L}$ ).

\section{Conclusions}

ALNR is one of the major factors for locally advanced TESCC after radical surgery. Although many factors may affect ALNR, including tumor location, width of tumor, T stage, N stage, TNM stage, ratio of LNM, vessel carcinoma embolus, cancerous nodes, LNM in the middle and lower mediastinal region, $\mathrm{LNM}$ in the abdominal region and the ratio of LNM in the abdominal region, only LNM in the abdominal region was the independent factor. Therefore, positive pathological abdominal LNs should be the major indication for abdominal irradiation in the PORT of TESCC. We recommended that the target volume for PORT in the abdominal area includes station 8 , station 9, station $16 \mathrm{a} 2$ and station $16 \mathrm{~b} 1$ and proposed a specific delineation of the clinical target volume based on the distribution of ALNR on template CT images. More studies with a larger sample are needed to verify our results.

\section{Abbreviations}

AA, abdominal aorta; ALNR, abdominal lymph node recurrence; $\mathrm{CA}$, celiac artery; $\mathrm{CHA}$, common hepatic artery; CT, computer tomography; EC, esophageal cancer; IVC, inferior vena cava; LNs, lymph nodes; LNM, lymph node metastasis; LRV, left renal vein; MR, magnetic resonance; SLN, sentinel lymph nodes; PORT, postoperative radiotherapy; TEC, thoracic esophageal cancer; TESCC, thoracic esophageal squamous cell cancer.

\section{Data Sharing Statement}

The datasets used and/or analyzed during the current study are available from the corresponding author on reasonable request.

\section{Ethics Approval}

Data collection and analysis were approved by the institutional review board of the First Affiliated Hospital of Anhui Medical University. Informed consent was waived due to its retrospective design and no identifiable information was disclosed. This study was conducted in accordance with the Declaration of Helsinki.

\section{Consent for Publication}

All the authors have consented to the publication of this manuscript.

\section{Acknowledgments}

We thank Jie Wang MD (Department of Medical Imaging, the First Affiliated Hospital of Anhui Medical University, Hefei, 230022, Anhui, P. R. China) for the acquisition and reading of $\mathrm{CT} / \mathrm{MR}$ image data. And thank for American Journal Experts' help in language modification for this article.

\section{Author Contributions}

All authors made substantial contributions to conception and design, acquisition of data, or analysis and interpretation of data; took part in drafting the article or revising it critically for important intellectual content; gave final approval of the version to be published; and agree to be accountable for all aspects of the work.

\section{Disclosure}

The authors declare that they have no competing interests.

\section{References}

1. Smyth EC, Lagergren J, Fitzgerald RC, et al. Oesophageal cancer. Nat Rev Dis Primers. 2017;3(1):17048. doi:10.1038/nrdp.2017.48

2. Wong AT, Shao M, Rineer J, Lee A, Schwartz D, Schreiber D. The impact of adjuvant postoperative radiation therapy and chemotherapy on survival after esophagectomy for esophageal carcinoma. Ann Surg. 2017;265(6):1146-1151. doi:10.1097/SLA.0000000000001825

3. Yu J, Ouyang W, Li Y, et al. Value of radiotherapy in addition to esophagectomy for stage II and III thoracic esophageal squamous cell carcinoma: analysis of surveillance, epidemiology, and end results database. Cancer Med. 2019;8(1):21-27. doi:10.1002/cam4.1731

4. Malhotra GK, Yanala U, Ravipati A, Follet M, Vijayakumar M, Are C. Global trends in esophageal cancer. J Surg Oncol. 2017;115 (5):564-579. doi:10.1002/jso.24592

5. Kang J, Chang JY, Sun X, Men Y, Zeng H, Hui Z. Role of postoperative concurrent chemoradiotherapy for esophageal carcinoma: a meta-analysis of 2165 patients. J Cancer. 2018;9(3):584-593. doi: $10.7150 /$ jca. 20940

6. Lv J, Cao XF, Zhu B, Ji L, Tao L, Wang DD. Long-term efficacy of perioperative chemoradiotherapy on esophageal squamous cell carcinoma. World J Gastroenterol. 2010;16(13):1649-1654. doi:10.3748/ wjg.v16.i13.1649

7. Wang Y, Zhu L, Xia W, Wang F. Anatomy of lymphatic drainage of the esophagus and lymph node metastasis of thoracic esophageal cancer. Cancer Manag Res. 2018;10:6295-6303. doi:10.2147/ CMAR.S182436

8. Wang Y, Zhang L, Ye D, et al. A retrospective study of pattern of recurrence after radical surgery for thoracic esophageal carcinoma with or without postoperative radiotherapy. Oncol Lett. 2018;15 (3):4033-4039. doi:10.3892/ol.2018.7807

9. Wang Y, Zhu L, Xia W, Wu L, Wang F. The impact of adjuvant therapies on patient survival and the recurrence patterns for resected stage IIa-IVa lower thoracic oesophageal squamous cell carcinoma. World $J$ Surg Oncol. 2018;16(1):216. doi:10.1186/s12957-018-1516-1

10. Zhang X, Yang X, Ni J, et al. Recommendation for the definition of postoperative radiotherapy target volume based on a pooled analysis of patterns of failure after radical surgery among patients with thoracic esophageal squamous cell carcinoma. Radiat Oncol. 2018;13 (1):255. doi:10.1186/s13014-018-1199-3 
11. Yu J, Ouyang W, Li C, et al. Mapping patterns of metastatic lymph nodes for postoperative radiotherapy in thoracic esophageal squamous cell carcinoma: a recommendation for clinical target volume definition. BMC Cancer. 2019;19(1):927. doi:10.1186/s12885-019-6065-7

12. Chen J, Cai W, Lin Y, et al. Patterns and rates of abdominal lymphatic metastasis following esophageal carcinoma. PLoS One. 2017;12 (10):e0185424. doi:10.1371/journal.pone.0185424

13. Sano T, Aiko T. New Japanese classifications and treatment guidelines for gastric cancer: revision concepts and major revised points Gastric Cancer. 2011;14(2):97-100. doi:10.1007/s10120-011-0040-6

14. Hsu PK, Huang CS, Wang BY, Wu YC, Hsu WH. Survival benefits of postoperative chemoradiation for lymph node-positive esophageal squamous cell carcinoma. Ann Thorac Surg. 2014;97(5):1734-1741. doi:10.1016/j.athoracsur.2013.12.041

15. Hwang JY, Chen HS, Hsu PK, et al. A propensity-matched analysis comparing survival after esophagectomy followed by adjuvant Chemoradiation to surgery alone for esophageal squamous cell carcinoma. Ann Surg. 2016;264(1):100-106. doi:10.1097/SLA.0000 000000001410

16. Yu S, Zhang W, Ni W, et al. A propensity-score matching analysis comparing long-term survival of surgery alone and postoperative treatment for patients in node positive or stage III esophageal squamous cell carcinoma after R0 esophagectomy. Radiother Oncol. 2019;140:159-166. doi:10.1016/j.radonc.2019.06.020
17. Takeuchi H, Fujii H, Ando N, et al. Validation study of radio-guided sentinel lymph node navigation in esophageal cancer. Ann Surg. 2009;249(5):757-763. doi:10.1097/SLA.0b013e3181a38e89

18. Xiao ZF, Yang ZY, Liang J, et al. Value of radiotherapy after radical surgery for esophageal carcinoma: a report of 495 patients. Ann Thorac Surg. 2003;75(2):331-336. doi:10.1016/S0003-4975(02)04401-6

19. Ni W, Chen J, Xiao Z, et al. Adjuvant radiotherapy for stage pN1M0 esophageal squamous cell carcinoma: results from a Chinese twocenter study. Thorac Cancer. 2019;10(6):1431-1440. doi:10.1111/ 1759-7714.13088

20. Chen Y, Hao D, Wu X, et al. Neoadjuvant versus adjuvant chemoradiation for stage II-III esophageal squamous cell carcinoma: a single institution experience. Dis Esophagus. 2017;30:1-7. doi:10.1093/ dote/dox016

21. Skandalakis JE, Skandalakis LJ, Skandalakis PN. Anatomy of the lymphatics. Surg Oncol Clin N Am. 2007;16(1):1-16. doi:10.1016/j. soc.2006.10.006

22. Haijun Y, Qiuji W, Zhenming F, et al. A new approach to delineating lymph node target volumes for post-operative radiotherapy in gastric cancer: a phase II trial. Radiother Oncol. 2015;116(2):245-251. doi:10.1016/j.radonc.2015.07.010

\section{Publish your work in this journal}

Cancer Management and Research is an international, peer-reviewed open access journal focusing on cancer research and the optimal use of preventative and integrated treatment interventions to achieve improved outcomes, enhanced survival and quality of life for the cancer patient.
The manuscript management system is completely online and includes a very quick and fair peer-review system, which is all easy to use. Visit http://www.dovepress.com/testimonials.php to read real quotes from published authors. 